\title{
Smart Farm Assist Robot
}

\author{
Nitin Krishna V, Ragunath B, Kowshika Priya B, Sivaranjani M, Vasanthamani K
}

\begin{abstract}
Autonomy in agriculture is the need of the hour in today's world. Advancements in technology have made it possible to design autonomous systems that carry out their intended operations efficiently, without any human intervention. However, most farmers still carry out agricultural operations manually using simple and conventional tools like a wooden plough, sickle, etc. Large-scale mechanization and autonomous systems are affordable only by medium and large class farmers who possess more than 2.00 hectares of agricultural land. Marginal and small-class farmers find it difficult in managing the workforce at an affordable cost. A user-friendly cost-effective approach will be a valuable support system for this sector. This paper proposes a novel design of a seed sowing robot with two operating modes; manual control by the operator and remote operation through GPS. The proposed seed sowing bot extracts the features of the agricultural field under consideration and adopts the optimal speed for seed sowing. Parameters like temperature, humidity, and soil moisture, which are pivotal in carrying out agricultural operations are measured by the use of different sensors embedded in the robot. Arduino ATMEGA2560 controls the locomotion of the bot and Raspberry $P i$ is used for image classification and obstacle detection. Sunset and the presence of rain are detected and the corresponding feasible actions are programmed to be followed by the robot automatically. A user-friendly mobile application has been developed to issue commands to the robot. The robot intends to reduce human efforts and provide intelligent aid to marginal and small class farmers while being affordable.
\end{abstract}

Keywords: Agribot, Autonomous agriculture, Smart farming, Seed sowing robot.

\section{INTRODUCTION}

A griculture plays a tremendous and pivotal role in the economy of any country. It contributes to the development of a country's economy by providing food and raw materials to other sectors and creates a demand for goods produced in non-agricultural sectors. Improvements in agricultural methods and land use become essential for improving food security and attaining sustainable development. It is the main source of income, food, and employment for country-dwellers. Based on the operational holdings, the farmers are categorized into four classes namely small,

Revised Manuscript Received on October 20, 2020.

* Correspondence Author

Nitin Krishna V*, Department of ECE,PSG College of Technology, Coimbatore, India. Email: nitinkrishna.cbe@gmail.com

Ragunath B, Department of ECE, PSG College of Technology, Coimbatore, India. Email: raghunathbalakrishnan3@gmail.com

Kowshika Priya B, Department of ECE, PSG College of Technology, Coimbatore, India. Email: kowshikapriya1005@gmail.com

Sivaranjani M, Department of ECE, PSG College of Technology, Coimbatore, India. Email:sivaranjani0831@gmail.com

Vasanthamani K, Department of ECE, PSG College of Technology, Coimbatore, India. Email:kvm.ece@psgtech.ac.in

(C) The Authors. Published by Blue Eyes Intelligence Engineering and Sciences Publication (BEIESP). This is an open access article under the CC BY-NC-ND license (http://creativecommons.org/licenses/by-nc-nd/4.0/) marginal, medium, and large classes. Small class farmers have less than a hectare of land. Marginal class farmers possess lands of size between 1.0 and 2.0 hectares. Medium and large class farmers operate in lands ranging between 2.0 to 10.0 hectares and more than 10.0 hectares. In India, the agricultural census of 2015 reveals that the percentage of small and large class farmers in the country is $86.2 \%[1]$. However, the majority of the farmers in this sector are not affordable to invest in modernized machinery to improve their yield. Advancements in science and technology, including sensors, machines, devices and, information technology have enabled farmers to deviate from the conventional agricultural practices. Precision agriculture helps farmers to make agriculture more efficient and profitable. Autonomous robotic systems assist farmers in increasing crop productivity, reducing the use of water, fertilizers, and pesticides, and to face the alarming decline in the number of agricultural labors all over the globe[2]. Furthermore, the innovative technologies ensure a reduced impact on natural ecosystems and growing conditions, thus paving the way for a better environment and safer foods. Robotic farm swarms, variable-rate swath control, agricultural robots, and equipment telematics are few of the effective and promising technologies in agriculture. Driverless tractors, drones, and robots are being used more widely than ever. Fertilizers, Pesticides, agricultural labors and availability of water are the factors that influence the production cost and yield in agriculture. So, these machines and devices become ideal choices in Smart farming to effectively make use of these resources. In this paper, a smart farm assist robot has is proposed. This robot primarily focuses on seed sowing in such a way that the land is utilized effectively. Theproposed robot is user friendly and can be operated manually or autonomously. The bot is designed to sow seeds with even space and uniformity by looking into the characteristics of the land. When operated in autonomous mode, a convolutional neural network-based image classification model extracts the feature of the farming land and fixes the optimum speed for seed dropping and movement. Section II of this paper presents the literature survey done on related works and similar agricultural robots. Section III describes the methodology adopted by the robot. Section IV illustrates detailed description of the design and implementation of subsystems of the bot. The construction, electronic components used, the application developed and image classification model used are explained in this section. In section $\mathrm{V}$, the experimental results are presented. In section VI and VII, the future work and conclusion is presented.

Published By:

Blue Eyes Intelligence Engineering DOI:10.35940/ijeat.A1904.1010120

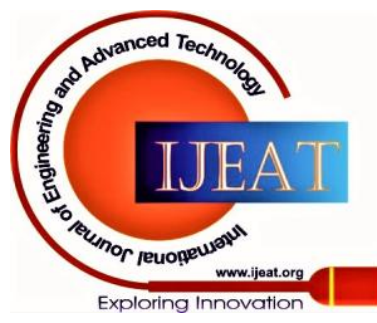




\section{Smart Farm Assist Robot}

\section{RELATED WORK}

An Ag Ro-Bothas been designed for multiple agricultural processes like digging the soil, seed sowing, harvesting, and irrigation. Solar panels have been used in thatAgRo-Bot to recharge the batteries. The system has a cutter in the front frame, a robotic arm with metallic teeth for digging the soil, a funnel to store the seeds, a sprinkler for irrigation, and a solar panel for recharging the batteries. The project has been implemented using the Arduino board ATMEGA 328p[3]. An agriculture assist robot has been designed for seeding and fertilizing. A network of sensors is used for measuring $\mathrm{pH}$ value, moisture, and temperature of the soil. The system uses Raspberry Pi for live streaming of the field for obstacle detection using obstacle detecting sensors. The system has two main sections, robot end, and control section, intercommunicated by internet communication technologies. Arduino Atmega328 is the controller used as the micro-controller[4]. The need for agro-bots, history of agro-bots, recent trends, and technologies adopted in the design and implementation of agro-robots has been described[5]. An agribothas been designedfor performing elementary functions like weeding of the field, sowing, and obstacle detection. It is designed in such a way to sow any seeds in the seed dispenser. The project was implemented agricultural robotwas designed to perform multiple purposes like ploughing, sowing, levelling, irrigation, and webcam surveillance to know the condition of the soil. This system uses GPS and magnetometer for heading towards the specific coordinates in a feasible direction. It also uses the GSM module to send messages to the user. The bot is implemented by using ATMEGA AT328p microcontroller[7]. Another robot has been modelled to perform agricultural functions like ploughing, seeding and irrigation. This system consists of a cultivator to dig the soil, a shaft to sow the seeds, a leveller to close the seeds and a water pump sprayer for irrigation purposes. The entire mechanism of this system is controlled using a Bluetooth module and Android application installed in the smartphone. AT89S52 microcontroller is used to control the entire system[8]. A multifunction robot has been designed for several agricultural farming processes like hole digging, seed dispensing, hole filling, and drip irrigation. The system consists of a toothed wheel for drilling holes as the wheels rotate. The hole is filled by using a glass fibre sheet attached to the backside of the bot. It has a seed storage tank that has a load cell to monitor the status of the tank[9]. A bot consisting of 2 main units; Handheld transmitter unit for communication and Sowing mechanism for seeding. This system consists of PIC Microcontroller interfaced with Encoder HT12E, Decoder HT12D, and receiver RX433 for data transmission[10]. Another agricultural bot has been modelled to perform basic farming processes like planting, harvesting, and pesticide spraying. The system consists of a camera employed for image processing. ATMEGA2560 is the master microcontroller, ATMEGA8 acts as a slave microcontroller with inbuilt sensors, gripper \& camera arrangement, indicators like a diode, LCD, etc[11]. Another robot to sow the seeds, cut the covering the seeds. The system has an ultrasonic sensor for type of seed and LDR is used for indicating the number of using Arm cortex SAM 3X8E[6]. An autonomous

grass, and spray pesticides is designed. The whole system is powered by a solar panel and has a funnel to store the seeds. The system has rotating blades to cut the grass and a slider to sow the seeds. The system is operated through the Android Application. Arduino Atmega328 Microcontroller is used in this project[12].

\section{METHODOLOGY}

The user is given an option to select the mode of operation of the bot during the power uptime using the Farm assist android application. In manual mode, the bot's movement can be controlled using the navigation keys in the app. The seed dropping mechanism, bot's speed, and the seed dropping rate can be configured in the manual mode. In autonomous mode, the GPS coordinates of the area to be sown are fed to the bot through the mobile app. Initially, the bot goes to the first-way point and then all sensors are activated. Image classification and field state extraction are done to calculate the depth of ploughing. Based on this, the speed of the bot, the rate at which the seeds are dropped is calculated concerning the terrain and adopted by the bot. Adapting based on the state of the field helps in carrying out the operations efficiently. The bot moves along the defined path simultaneously sowing the seeds. The quantity of seeds in the container is monitored constantly through the IR sensor and if the quantity reduces below the fixed threshold level, the bot is moved to the home location to refill the seeds. After the seeds are filled, the bot returns to the location where it stopped sowing and resumes its operation. Obstacle detection and avoidance are carried out using the ultrasonic sensor and the seed sowing bot alters its path in case of an obstacle. Rain sensor is used to detect the rain and bot returns to the home location in case of rain. This helps in preventing the bot from getting stuck in a quagmire and protects the water-sensitive components in the bot. When the light intensity falls below a threshold value, the LED lamps are turned on through the relay to provide sufficient lighting to the camera. The block diagram of the methodology followed is shown in the Fig. 1.

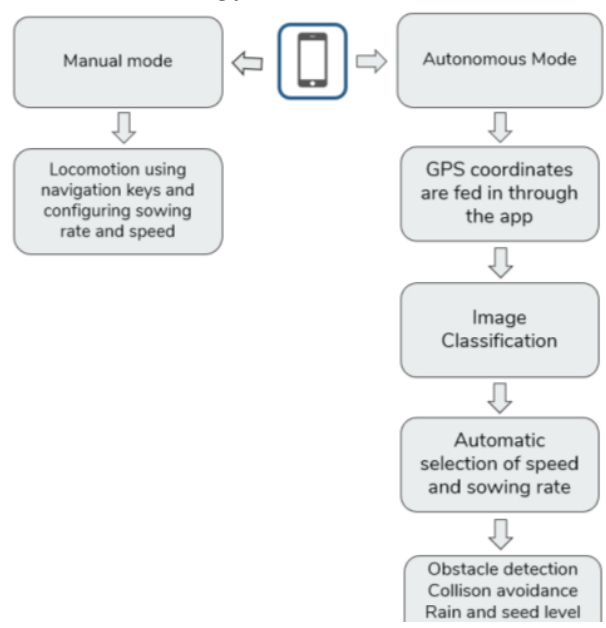

Fig. 1. Block Diagram of the working mechanism

\section{DESIGN AND IMPLEMENTATION}

Published By:

Blue Eyes Intelligence Engineering

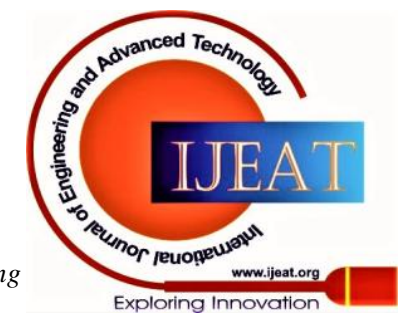




\section{A. Mechanical construction}

The seed sowing bot is designed in such a way that its center of gravity remains constant in the middle to ensure stability during motion by even distribution of weight over the entire base. Furthermore, this ensures that all the dc gear motors are required to provide the same amount torque.

Adopting a four-wheel-drive ensures that the bot can move freely in rough terrains without being hindered or stopped by striations in the field. Aluminium is used as the base metal for the bot due to its durability, strength, density, and flexibility. Seeds are stored in an acrylic container. Acrylic material is selected owing to its lightweight, high tensile strength and transparency. The seed container is designed in a funnel-like shape to direct the seeds downwards and the capacity of the container is five to six kg of seed (depending on seed size). A metal slider attached to a dc motor beneath the base of the bot is used to control the flow of seeds through the pipes running through holes on the base. The diameter of the pipes and the distance between two adjacent pipes can be altered manually depending on the seed to be sowed. The feature extraction of the agricultural field is performed using the panoramic image captured by the camera. The camera is fixed in the front on the shaft attached to the servo motor which facilitates the rotation of the camera in both clockwise and anti-clockwise direction. Two ultrasonic sensors along with the LED lights aids obstacle detection, obstacle avoidance, and collisionavoidance. The magnetometer is isolated using a separate acrylic pipe as the magnetometer is sensitive to electromagnetic interferences. The orientation of the magnetometer is fixed by repeatedly calibrating it to match the earth's magnetic field. The electronic assembly is fixed in the front of the bot and is insulated properly for protecting from electrical and environmental conditions. The DHT-22 sensor and rain sensor is fixed such that both are exposed to open air. The CAD sketch of the bot has been shown in the Fig. 2. The base of the bot has been shown in the Fig. 3. A $12 \mathrm{~V}$ solar panel is mounted as shown in the Fig. 4 to recharge the battery.

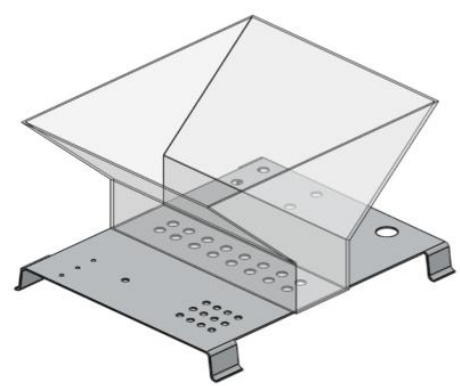

Fig. 2.CAD Sketch of the base of the bot

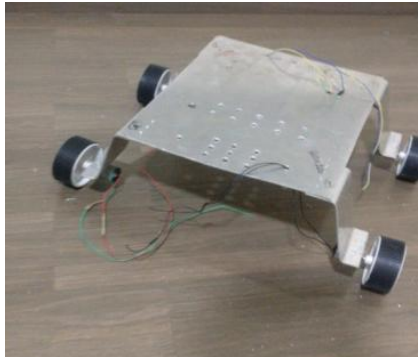

Fig. 3.The aluminium metal base

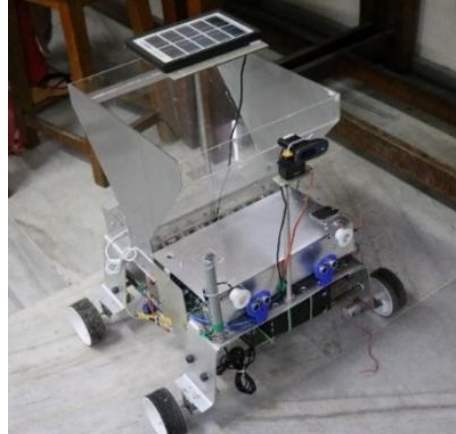

Fig. 4.Bot with solar panel

\section{B. Electronic System Design}

Arduino Mega is used to controlling the entire locomotion of the seed sowing bot. The DC gear motors are connected to the Arduino board through L298N motor drivers. The magnetometer HMC 5883L and GPS module Neo 6M are interfaced through the UART interface. Soil hygrometer, rain, DHT22, light, IR sensors are also interfaced to the Arduino Mega. The communication between the mobile app and the Arduino Mega is implement using HC05 Bluetooth module. Raspberry Pi is used for image classification and obstacle detection. Raspberry $\mathrm{Pi}$ is used to handle high computational speed and powerful processing for neural network modelling. Raspberry $\mathrm{Pi}$ serially transfers the processed information to the Arduino board. A Magnetometer, which is a 3-axis digital compass coupled along with the GPS module makes sure that the bot moves to the desired coordinates. The bot covers the desired portion of the field while maintaining the boundaries with the help of the GPS module. A soil hygrometer is used to measure the moisture content of the soil, while humidity and temperature sensor provides the temperature, humidity, and heat index of the agricultural land. The block diagram of the electronic system is shown in Fig. 5.

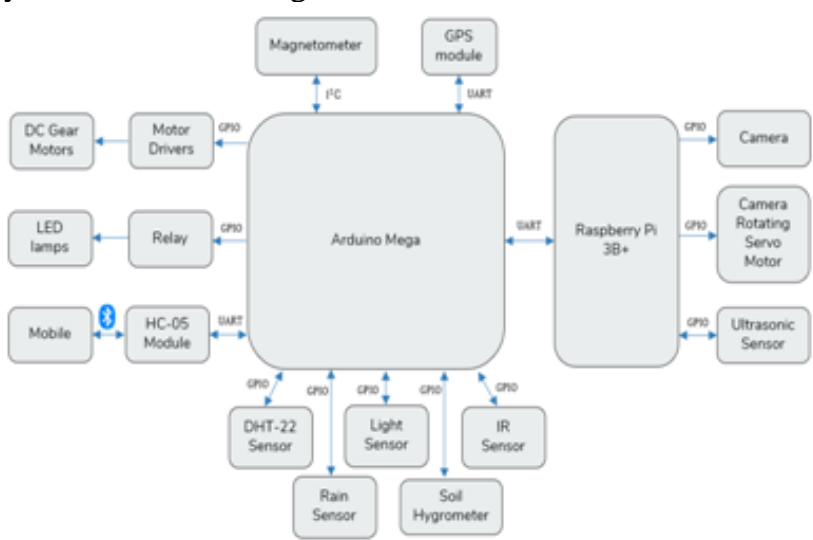

Fig. 5.Block Diagram of Electronic system

\section{App GUI Design - Smart Farm Assist App}

MIT App inventor is used to design the mobile application. The mobile app consists of GUI for the following controls.

1. To connect with the HC05 module by selecting it from the "Connect BT" list.

2. Navigation buttons as shown in the Fig. 6 namely "Forward", "Reverse", "Right 90", etc. aid the user in controlling the bot's locomotion manually.

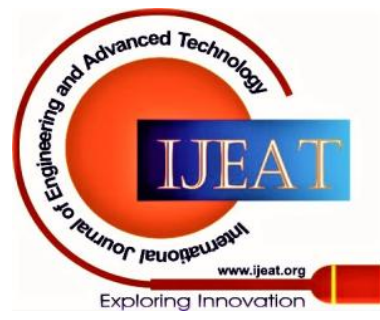


3. The speed of the bot can be increased by dragging the slider button to the right and can be decreased by dragging it to the left.

4. The LED lights can be switched on and off by pressing the "LIGHTS ON" and "LIGHTS OFF" buttons.

5. The slider - seed drop mechanism can be controlled by the buttons "SLIDER ON" and "SLIDER OFF", which initiate and stop the dropping of seeds.

6. The temperature, humidity, heat index and soil moisture content of the agricultural field is displayed in the "Notification window" as shown in Fig. 6 and Fig. 7.

7. The current coordinates of the bot can be obtained by pressing the "GPS LOCATION" button.

8. On pressing the "L, R, S" button indicates the status of sun light, rain and seed.

9. The autonomous mode of operation is initiated by pressing the "Go to waypoint" button. The waypoints are displayed in the Notification Window on pressing the "GPS info" button. The waypoints can be cleared by pressing the "Clear All" button. In case of any rain or shortage of seeds, the user is notified by alerts in the Notification Window".

The temperature and humidity are being displayed in the Notification Window on pressing the respective buttons as shown in Fig. 8 and Fig. 9.
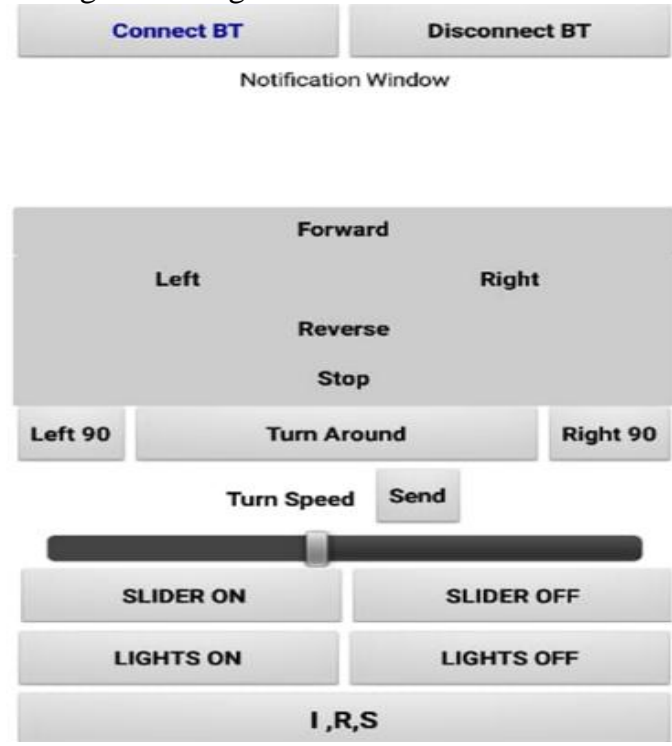

Fig. 6.Navigation and Sowing Control screen of Farm Assist

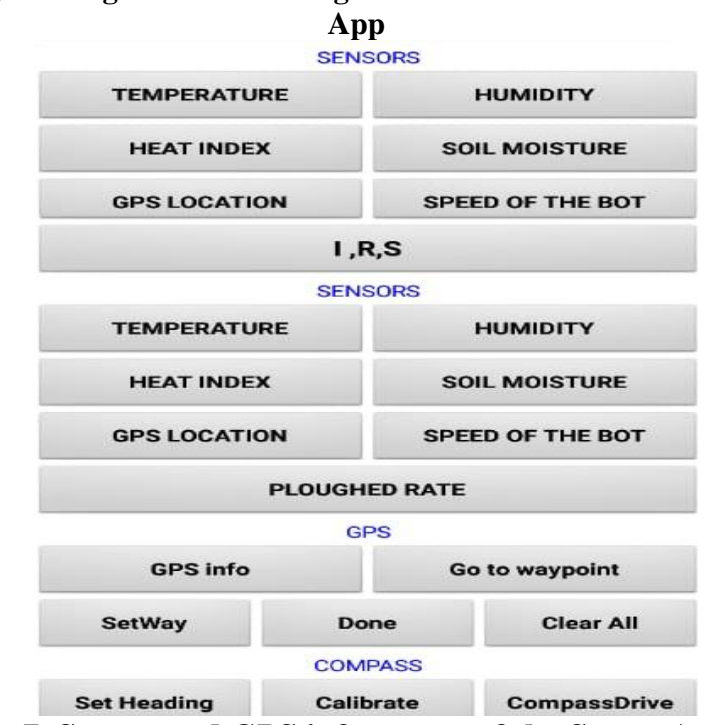

Fig. 7. Sensor and GPS info screen of the Smart Assist App

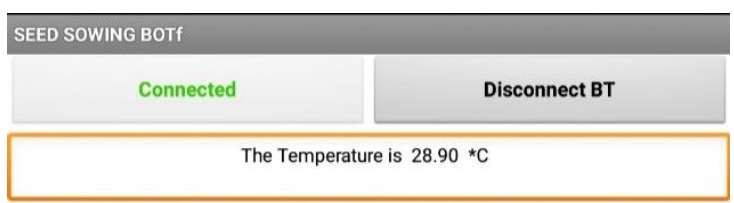

Fig. 8.The temperature being displayed on pressing the TEMPERATURE button

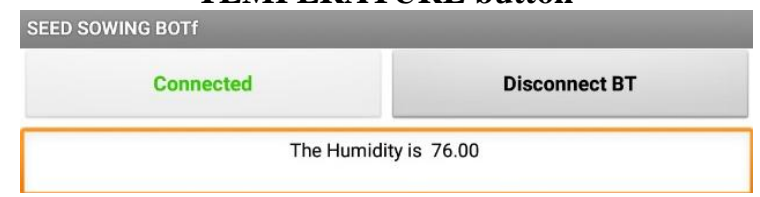

Fig. 9.The humidity being displayed on pressing the HUMIDITY button

\section{Image Classification Using Convolutional Neural Networks (CNN)}

A Convolutional Neural Network (CNN) is the advanced method to extract higher representations of the image. CNN takes the raw data of the raw image as input and it automatically decides the type of object based on the extracted features, texture and shape. A three-dimensional matrix is received by $\mathrm{CNN}$ as an input feature map. The length and width of the images in pixels correspond to the size of the first two dimensions. The third dimension corresponds to the three channels of color information (red, green, blue). There are three operations performed by each of the stacks of modules present in CNN.

- Convolution

- ReLU

- Pooling

The input layer consists of the raw pixel values of the image while the convolution layer computes the output of each neuron which is connected to the region of the input layer. Convolution layer computes the dot product between the weight of neurons, and a small region they are connected to in the input layer.

The ReLU (Rectified Linear A) layer will apply element-wise activation function such as $\max (0, x)$ and the down sampling operation along the spatial dimensions (height, width) is performed by the pooling layer.

- INPUT [32x32x3] holds the raw pixel values of the image, in this case the image is of width 32 , height 32, and with three color channels R,G,B.

- The RELU layer applies an elementwise activation function, such as the $\max (0, \mathrm{x})$ thresholding at zero leaving the size of the volume unchanged.

- $\quad$ The POOL layer performs a down sampling operation along the spatial dimensions i.e, the height and width producing a result in volume such as [16x16x12].

The Image classification model as shown in Fig. 10 is developed using transfer learning to determine the ploughing rate for the field. The transfer learning mechanism is used here to avoid latency as it utilizes the pre-trained neural network. If the training of the neural network is done from scratch, it takes a longer period of time. The image recognition model has two parts: Feature extraction part with a convolutional neural network.

Published By:

Blue Eyes Intelligence Engineering

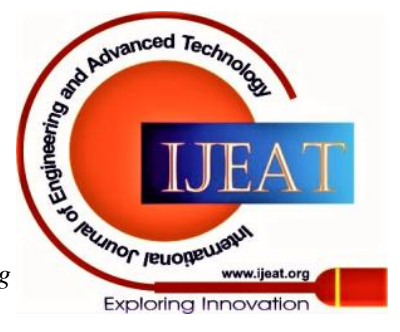


In transfer learning, when a new model is built to classify the dataset, one tends to reuse the feature extraction part and re-train the classification part with the required dataset. The categories of images are stored in the form of a text file. This file is used to translate labels into internal ID numbers in further processes such as ploughed $=0$, unploughed $=1$, not a farming field $=2$, etc. The images in the data set are randomly split into a training set with $90 \%$ data and an evaluation set with $10 \%$ data.

Conv. Module\#1 Conv. Module \#2 Classification

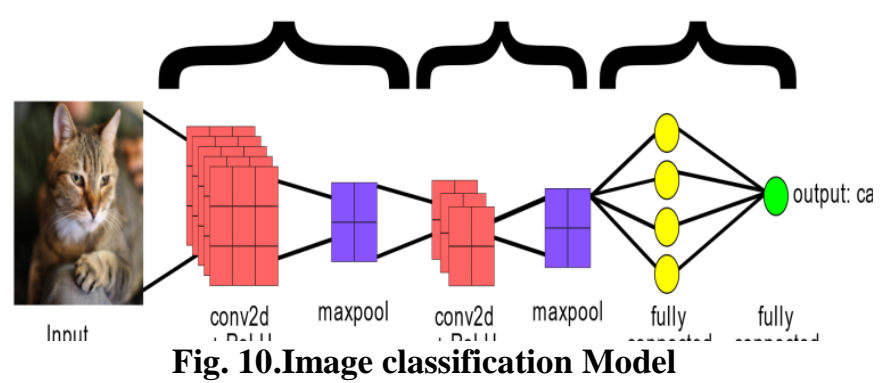

PROCESS OF TRAINING THE MODEL:

The image recognition model consists of two parts:

- $\quad$ Feature extraction using CNN.

- Classification part with fully connected and Softmax layers.

The flowchart of image recognition model is shown in Fig. 11. The development of the feature extraction part is the complex part of the image recognition model. Thus, when a new model is developed to classify images, the feature extraction part of the Inception-v3 model is retained and the classification part is retrained with the data set containing the images of the agricultural field.

The image classification model that hasbeen developed for the Autonomous bot consists of 2400 images with three categories with 800 images in each category (the more the number of images in each category, better will be the classification results) and are mutually exclusive and there is no overlap between them. This classification model uses 2400 images so that it is equally distributed among the three categories to be classified. Each category can contain $2 * * 27$ - 1 images. While training, the images with simple distortions including crops, scales, and flips, the results can beimproved and these kinds of testing reflect the kind of variations expected in the real world, and so can help train the model to cope with natural data more effectively.

The images are given as input to the feature extraction part of Inception-v3 which converts the image data into feature vectors for each image. A feature vector is just a vector that contains information describing an object's important characteristics. A feature vector represents the features of the image in an abstract manner. The classification of images can be done easily with the vector values rather than raw image data. The next step involved is to pre-process the images as shown in Fig. 13. The steps involved in pre-processing the images are:

- Read the image

- $\quad$ Resize the image

\section{- Remove noise}

- $\quad$ Smoothing edges

The steps involved in resizing the image are cropping and scaling In the full image, the bounding box is placed to perform the cropping. The size of the bounding box relative to the input image is controlled by the cropping parameter. If the cropping parameter is zero, then no cropping is performed. If the cropping parameter is $50 \%$, then the size of the bounding box will be half the width and height of the input.

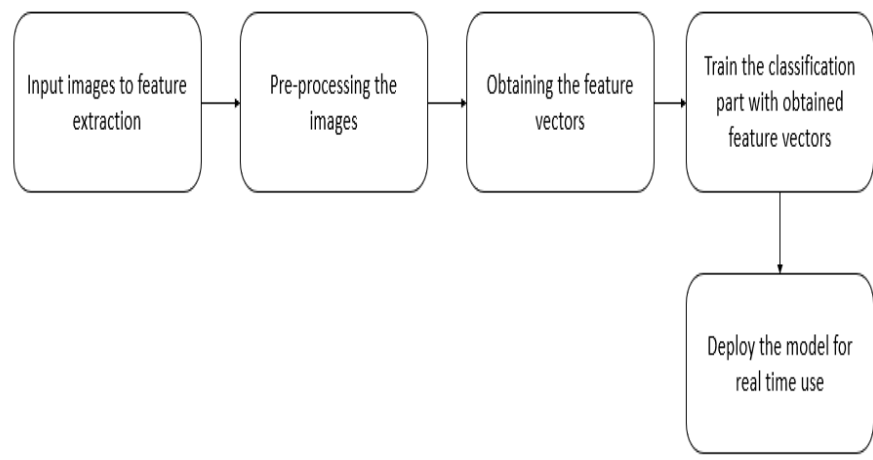

Fig. 11.Flowchart for training the image recognition model

Scaling is similar to cropping in which the size of the bounding box varies randomly and it is always centered in the full image as shown in Fig. 12.

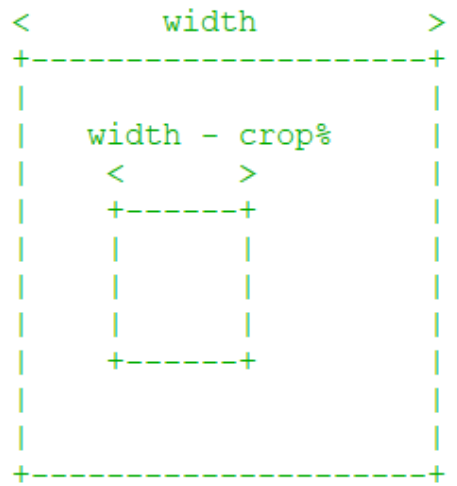

Fig. 12.Scaling of the field

Args:

flip_left_right: Boolean value to randomly mirror images horizontally.

random_crop: Integer percentage setting of the total margin used around the crop box.

random_scale: Integer percentage to vary the scale by.

random_brightness: Range of integers to multiply the pixel values by the graph.

input_width: Horizontal size

input_height: Vertical size

input_depth: Number of channels the expected input image should have.

input_mean: Pixel value that should be zero

input_std: Tells how much to divide the pixel values by before recognition.

Published By:

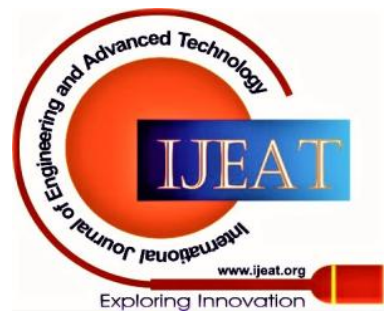




\section{Deployment of model:}

When the training is complete, the trained model is deployed for predictions. The output of the model consists of the name of the category to which the image belongs and the percentage to which the image belongs to that category.

PANORAMIC PICTURE USING IMAGE STITCHING:

Panorama image is constructed using cv2 and image processing techniques such as key point detection and local invariant descriptors, key point matching, RANSAC, and perspective warping are utilized. In order to obtain the panorama picture, there are four steps to be performed.

The first step involves the key points detection and local invariant descriptors extraction from the images. The second step is to match the descriptors between the two images. Using the matched feature vectors, the homography matrix can be is estimated using the RANSAC algorithm. The final step is applying the warping transformation with the help of the homography matrix obtained from the RANSAC algorithm.

The image stitching mechanism requires only one parameter. It requires only the images to be stitched. The key points and the local invariant descriptors (i.e SIFT) are extracted from the image when it is unpacked

To stitch image,cv2.wrap Perspective requires three arguments: The image that is to be wrapped, the $3 x$ 3 transformation matrix $(\mathrm{H})$, and finally the shape out of the output image. The width of the output image is obtained by taking the sum of the widths of both images and the height is of the output is the height of the second image.

The match Keypoints function requires four arguments. They are the test variable of David Lowe's ratio, the RANSAC re-projection threshold value, the key points, and the feature vectors from both the images.

The two images Fig. 14 and Fig. 15 are fed as input to the 16.

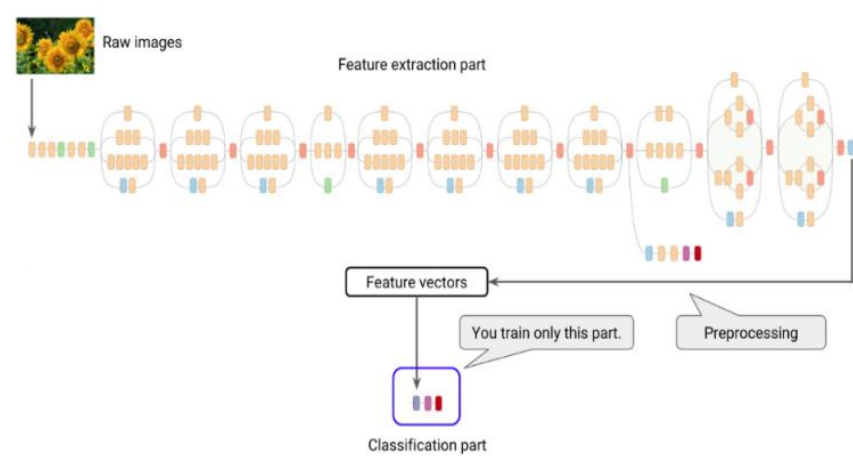

Fig. 13.Relationship between the pre-processing and the training

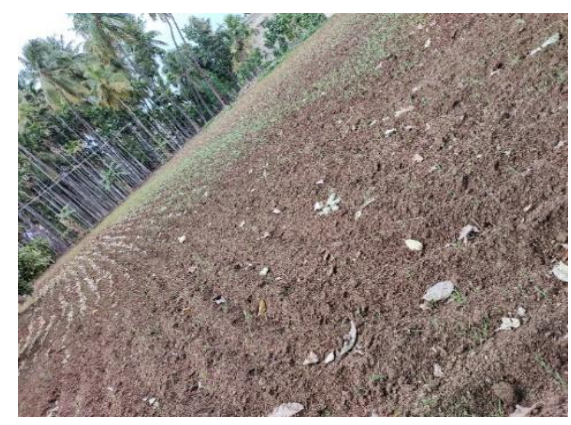

Fig. 14. One view of the field image stitching package to obtain the output shown in Fig.
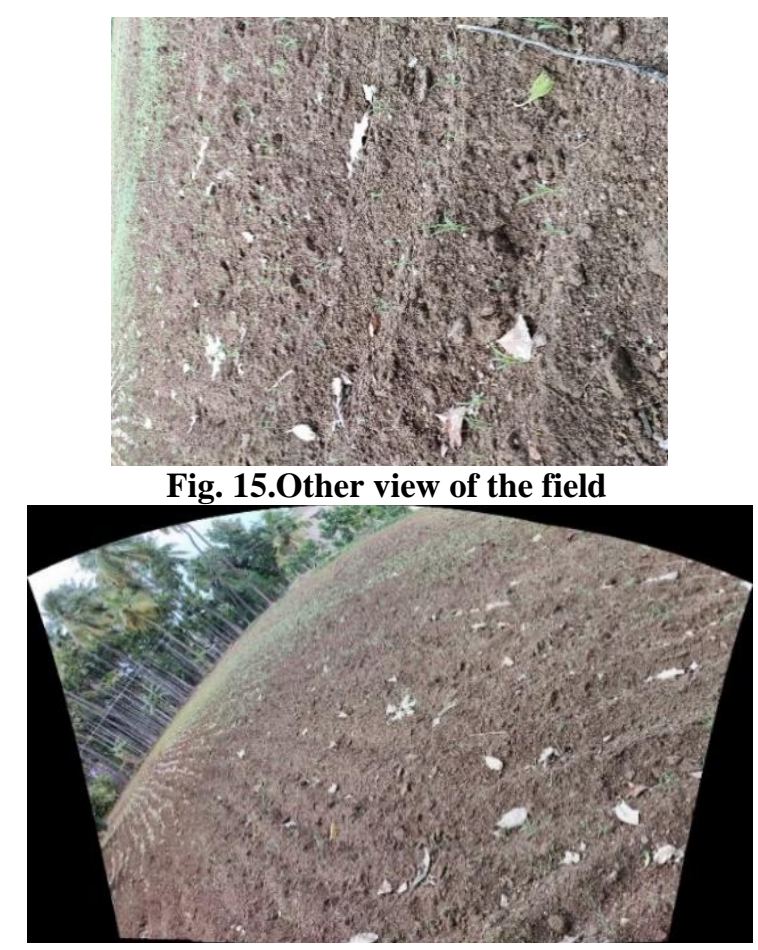

Fig. 16.The stitched Image

\section{RESULTS AND DISCUSSION}

The bot was employed in an agricultural field which was moderately ploughed as shown in Fig. 14 and Fig. 15. The image classification model produced an output of 78.88 percent as shown in the Fig. 17 for the stitched image shown in the Fig. 16, thus providing the optimal speed and seed dropping rate to the bot. Four corn seeds of average diameter 1 inch separated by 3 inches were sown. It took about 45 minutes for the bot to cover an area of 1 square acre. The DC motors were able to provide enough torque for the bot to move in the field without any hindrance. The humidity of the agricultural field was $76 \mathrm{RH}$ as shown in Fig. 8,and the temperature of the field was $28.90^{\circ} \mathrm{C}$ asshown in Fig. 9. For fields where the model produces larger values, the speed of the bot and the seed dropping rate are decreased automatically. This ensures that the rows of seeds have the desired gap between them. The light intensity was continuously monitored. The two lamps turned on after the sunset. Similarly, the presence of rain was also monitored through the rain sensor. The solar panel was able to recharge the battery completely in about 5 hours. Table-I consolidates the experimental results obtained for the agricultural field shown in Fig, 17.

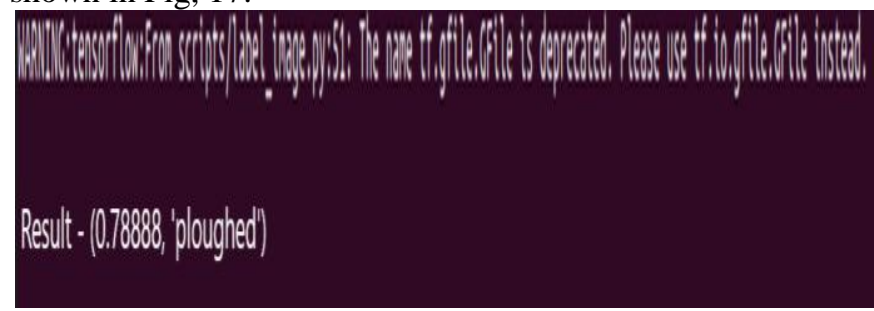

Fig. 17.Output of Image classification showing a match of 78 percent

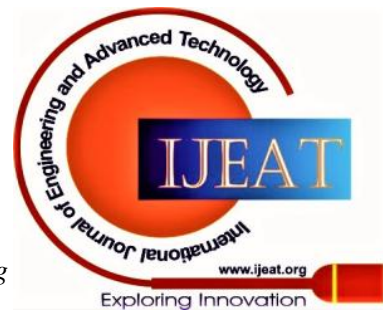


Table - I: Experimental results obtained for agricultural field shown in Fig. 17.

\begin{tabular}{|c|c|c|c|}
\hline $\begin{array}{c}\text { Output of } \\
\text { image } \\
\text { classification } \\
\text { model }\end{array}$ & $\begin{array}{c}\text { Time taken to } \\
\text { sow 1 square } \\
\text { acre of corn } \\
\text { seeds }\end{array}$ & Temperature & Humidity \\
\hline 0.788 & 45 minutes & $28.90^{\circ} \mathrm{C}$ & $76 \mathrm{RH}$ \\
\hline
\end{tabular}

\section{FUTURE SCOPE}

The bot can be modified to perform other agricultural operations like ploughing, weed control, fertilizer spraying, etc. The size of the bot can be made much larger to speed up the process. The mechanical structure of the bot can be strengthened by using tires that are more suited for agricultural terrains. This would increase the stability of the bot and ensure that the bot is able to more freely in field where the ploughed rate is very high. GSM module can be incorporated with the bot which would help notifying the user with important details through SMS. ALCD screen can be used in the bot to display sensor outputs. The Smart Farm Assist App can be made more user friendly, by using joystick for locomotion control in place of the existing buttons. The image classification model can be trained with more samples to produce more accurate results. Ultrasonic sensors with high range can be used to ensure that obstacles can be detected in advance. The features of this bot can used in the construction of drones, which can cover larger area within a short duration.

\section{CONCLUSION}

An autonomous seed sowing bot has been designed to carry out the process of sowing efficiently. The bot can either be operated in manual mode or autonomous mode. The adaptive nature of the bot concerning the environment allows the user to utilize the field to its maximum extent. The numerous sensors ensure that the bot performs its operations without any hindrance. The proposed design will overcome the numerous challenges associated with conventional agricultural operations. The seeds can be sown by the robot in evenly spaced rows with a considerable amount of uniformity. The modular structure of the robot provides ease for maintenance. The proposed device exhibits appreciable efficiency in terms of cost and power consumption, making it ideal for seed sowing. The proposed robot can help marginal and small-class farmers to carry out agricultural operations with less manual effort at an affordable cost.

\section{ACKNOWLEDGMENT}

Weexpress our gratitude to Mr. T. Venkatachalam, Assistant Professor, Dept of ECE, PSG College of technology for his exceptional support and enthusiasm without which this research paper would not have been possible.

\section{REFERENCES}

1. Varun B Krishnan (2018), What the agriculture census shows about land holdings in India. The Hindu, October 3, 2018.

2. Michell Zappa (2014), Emerging Agriculture Technologies That Will Change The World. Policy Horizons Canada, May 6, 2014.

3. Shubam Khandelwal, Neha Kaushik and Manoj K. R. Pandey (2017). "AgRo-Bot: An Autonomous Robot", International Journal of Advanced Research in Computer Science, Vol. 8, No. 5, May-June 2017.
4. ShivaprasadB. S., RavishankaraM. N., and Shoba B. N., (2014), "Design and Implementation of Seeding and Fertilizing Agriculture Robot", International Journal of Application or Innovation in Engineering \& Management, Vol. 3, Issue 6, June 2014.

5. Shavon McGlynn and Drew Walters (2019),"Agricultural Robots: Future Trends for Autonomous Farming", International Journal of Emerging Technologies and Innovative Research,Vol.6, Issue 4, April 2019.

6. Shraddha Muley (2017), "Robotic Vehicle for Seed Planting \& Weeding Applications", International Journal for Innovative Research in Science \& Technology, Vol 3, Issue 12, May 2017.

7. Shaik K., Prajwal E., Sujeshkumar B., Bonu M., and BalapanuriVamseedhar Reddy (2018), "GPS Based Autonomous Agricultural Robot",International Conference on Design Innovations for 3Cs Compute Communicate Control (ICDI3C), Bangalore, 2018, pp. 100-105.

8. Sowjanya K. D., Sindhu R., Parijatham M., Srikanth K., and Bhargav P., (2017), "Multipurpose autonomous agricultural robot", 2017International conference of Electronics, Communication and Aerospace Technology (ICECA), Coimbatore, 2017, pp. 696-699

9. Sweety Dutta, Udit Shanker, SulekhaKatiyar, Venktesh Singh, Mohd Nayab Zafar and Mohanta, J. C., (2019),"Development and Fabrication of an Autonomous Seed Sowing Robot”, 2019 IOP Conf. Ser.: Mater. Sci. Eng. 691012023.

10. AkshayNilawar, Pradnya P., Shilvant and Parmar Harpreet Kaur (2018), "Wireless Agricultural Seed Sowing Robot",International Journal of Latest Technology in Engineering, Management \& Applied Science (IJLTEMAS), Vol VII, Issue IV, April 2018, ISSN 2278-2540

11. Gowthami K., Greeshma K., and Supraja N., (2019),"Smart Farming Using Agri-Bot", International Journal of Applied Engineering Research,Vol 14, Issue 6, 2019 ISSN 0973-4562

12. Ranjitha B., Nikhitha M. N., Aruna K., Afreen K., and Venkatesh Murthy B. T., (2019), "Solar Powered Autonomous Multipurpose Agricultural Robot Using Bluetooth/Android App”,International Conference on Electronics Communication and Aerospace Technology [ICECA 2019], IEEE Xplore ISBN: 978-1-7281-0167-5.

\section{AUTHORS PROFILE}

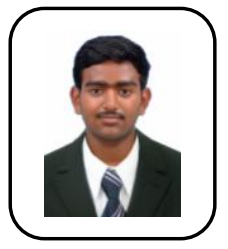

Nitin Krishna Vis a student of the department of Electronics and Communication Engineering in PSG College of Technology, Coimbatore. His interests are in VLSI, embedded systems design, and micro-electronics.He has been a part of numerous VLSI projects in the past. He has published two research papers in the design of digital circuits using reversible logic.

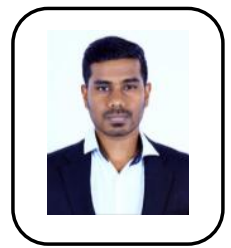

Ragunath Bis a student of the department of Electronics and Communication Engineering in PSG College of Technology, Coimbatore. He is interested in deep learning and artificial intelligence and has been a part of numerous projects pertaining to deep learning.

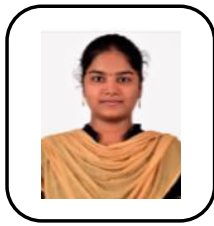

Kowshika Priya Bis a student of the department of Electronics and Communication Engineering in PSG College of Technology, Coimbatore. She is an enthusiast in developing innovative agricultural prototypes. Her interests include signal processing, embedded systems design, precision agriculture, and machine learning.

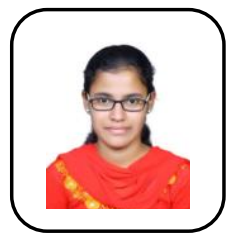

Sivaranjani Mis a student of the department of Electronics and Communication Engineering in PSG College of Technology, Coimbatore. She is interested in digital electronics and embedded systems. She has also completed projects pertaining to Application Programming Interface design in the past.

Published By:

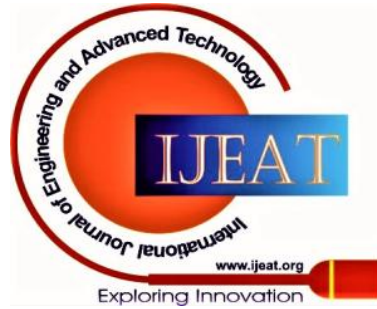




\section{Smart Farm Assist Robot}

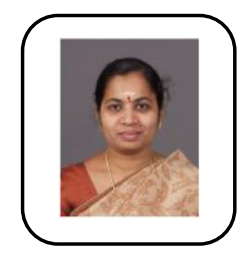

Vasanthamani Khas received a Masters in Applied Electronics from Bharathiar University,Coimbatore and Ph.D. in Information and Communication Engineering from Anna University.She has over 7 years of teaching experience in the Department of Electronics andCommunication Engineering in PSG College of Technology. She has around 10 publications inInternational and National journals and conferences. She was working in the design anddevelopment of electronic controllers for more than 17 years. The products developed includeBody Control Unit for Light Commercial Vehicle and Instrument clusters for two wheelers andfour wheelers for automotive sector, Production monitoring and control equipment for spinningmachine in textile industry, Power factor monitor and controller, Maximum demand monitor andcontroller. She has developed products using 8-bit, 16-bit and 32-bit processors.

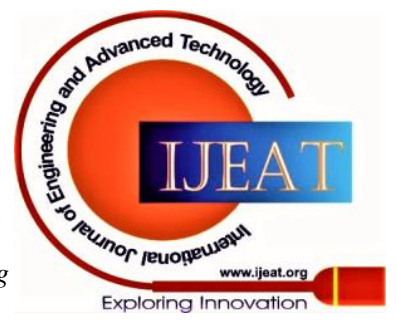

